\title{
Treino de Memória no Idoso Saudável: Benefícios e Mecanismos
}

\author{
Memory Training in Healthy Elders: Outcomes and Possible Mechanisms of Improvement
}

\author{
Mônica Sanches Yassuda*, Samila Sathler Tavares Batistoni, \\ Andréa Garofe Fortes, Anita Liberalesso Neri \\ Universidade Estadual de Campinas, Campinas, Brasil
}

\begin{abstract}
Resumo
Introdução: Pesquisas documentam declínio na memória ao longo do envelhecimento, entretanto, estudos apontam que o treino gera um aumento significativo no desempenho de idosos. Objetivo: Replicar resultados internacionais referentes a treino de memória com uma amostra brasileira de idosos saudáveis e investigar mecanismos, como o uso de estratégias, que possam explicar seus benefícios. Método: 69 idosos saudáveis, subdivididos em grupo experimental (GE) e grupo controle (GC), receberam quatro sessões de treino de memória (GC recebeu o treino após o pós-teste). O treino ofereceu informações sobre memória e envelhecimento, instrução e prática em organização de listas de supermercado e grifo de idéias principais em textos. Resultados: No pós-teste o GE apresentou melhor desempenho na recordação de texto e maior uso de estratégias (organização da lista e uso de grifo). Os dois grupos relataram menor número de queixas (medidas através do MAC-Q) e processamento de informações mais rápido (símbolos do WAIS-R) no pós-teste. Conclusões: Após o treino, os idosos fazem uso mais intenso de estratégias de memória, mas este fato não garante melhor desempenho (não houve aumento na memorização da lista). Os achados para a lista sugerem a presença da deficiência da utilização: os idosos usaram a estratégia, mas não se beneficiaram dela.

Palvaras-chave: Memória; treino; envelhecimento; estratégias.
\end{abstract}

\begin{abstract}
Introduction: Studies document a change in memory during the process of aging. However, studies report the possibility of improvement after training. Objective: To replicate international findings regarding the effects of memory training with a sample of Brazilian elders and to investigate mechanisms, such as the use of strategies, that can explain its benefits. Methods: 69 healthy Brazilian elders were randomly divided into an experimental group (EG) and a control group (CG), and received four sessions of training (CG received training after pos-test). Training included information on memory and the process of aging, instruction and practice in organizing grocery lists and the underlining of main ideas in short texts. Results: There was superior improvement for prose recall and greater use of strategies (organizing lists and underlining texts) for the EG at post-test. Both groups reported fewer memory complaints (assessed with the MAC-Q) and faster information processing (WAIS-R Digit Symbols sub-test). Conclusions: After training, elders intensify the use of strategies, however, this fact does not guarantee a better performance (there was no significant improvement in list recalls). List recall results suggest the presence of the utilization deficiency: elders used the strategy but failed to benefit from it.

Keywords: Memory; training; aging; strategies.
\end{abstract}

O estudo do envelhecimento cognitivo humano progrediu expressivamente nos últimos 50 anos. Sabe-se hoje que certos déficits de memória fazem parte do envelhecimento saudável. Encontram-se bem documentadas as diferenças entre adultos jovens e idosos em tarefas envolvendo a memória operacional e a memória episódica (Stuart-Hamilton, 2002; Yassuda, 2006). Quanto aos estágios do processo de memorização (codificação, estocagem, e resgate), sabe-se que existem diferenças significativas entre jovens e idosos durante a codificação e o resgate das informações (Kausler,

\footnotetext{
Endereço para correspondência: Escola de Artes, Ciências e Humanidades, Gerontologia, USP. Av. Arlindo Bettio, 1.000 - Ermelino Matarazzo, São Paulo/ SP - 03828-000 - Tel.: 011-3091-1029. E-mail: yassuda@usp.br

As autoras gostariam de agradecer a todos os voluntários que participaram da pesquisa, a FAPESP pelo apoio financeiro à pesquisa de pós-doutorado da primeira autora e a Darlene Fátima Brasileiro Corrêa e Mércia Barbosa Gomes Lopes que nos ajudaram durante a coleta de dados.
}

1991). Foi documentado ainda que com a idade o processamento das informações torna-se mais lento e dispendioso (Salthouse, 1991).

Entretanto, a literatura sugere que no envelhecimento saudável existe a possibilidade de compensação (pelo menos parcial) dos déficits cognitivos (Backman, 1989; Baltes \& Baltes, 1990; Dunlosky \& Hertzog, 1998). Pesquisas sobre treino cognitivo e de memória indicam que o idoso saudável é capaz de aproximar seu desempenho atual do seu desempenho máximo possível, revelando plasticidade cognitiva (Verhaeghen, 2000).

O estudo sobre a plasticidade cognitiva revela-se um campo fértil para a pesquisa básica, considerando que gera informações essenciais a respeito do envelhecimento humano. Ao mesmo tempo estes estudos têm implicações importantes para a prática clínica do profissional gerontólogo, 
pois a otimização da memória está relacionada à saúde, à autonomia e independência do idoso (Ramos, 2003).

Uma grande quantidade de estudos sobre treino de memória encontra-se disponível na literatura internacional. De maneira ampla, estes estudos podem ser sub-divididos em dois grandes grupos: estudos que ofereceram treino instantâneo específico para uma determinada tarefa de memória (e.g., Hulicka, \& Grossman, 1967, primeira publicação sobre treino; no Brasil, ver Lasca, 2003) e estudos que ofereceram treino extenso envolvendo diversas sessões com a duração total entre quatro e 15 horas (e.g., Zarit, Cole, \& Guider, 1981; no Brasil, ver Wood et al., 2000).

Os estudos de treino divergem não somente quanto à duração, mas em particular, quanto às estratégias ensinadas e à metodologia empregada, havendo na literatura grande diversidade quanto à magnitude dos efeitos, sua generalização para tarefas não treinadas e manutenção a longo prazo. Assim, os estudos foram agrupados e revisados em diversos artigos (por exemplo, Dunlosky, \& Hertzog, 1998) e em estudos meta-analíticos (Floyd \& Scogin, 1997; Verhaeghen, Marcoen, \& Goossens, 1992). Verhaeghen et al. (1992), analisaram a diferença no desempenho entre o pré e o pós-teste no grupo experimental, controle e placebo de 32 estudos de treino de memória episódica e documentaram que estes programas geram um aumento significativo no desempenho dos idosos. O efeito do treino é estatisticamente maior que o efeito de testes consecutivos (prática com testes que ocorre no grupo controle) e de programas de intervenção do tipo placebo (programas que não têm como objetivo o aperfeiçoamento da memória, como grupos de discussão). Esta meta-análise indicou que idosos aprendem a usar estratégias de memorização, mesmo as mais complexas, entretanto, não sugeriu a superioridade de nenhuma técnica em particular. Esta meta-análise corrobora a noção da plasticidade da memória episódica e sua importância como campo de estudo na gerontologia. Para uma revisão sobre os estudos de treino tradicionais ver Yassuda (2006).

Mais recentemente, metodologias inovadoras vêm sendo utilizadas nos estudos de treino, como o uso de vídeo cassete para ensinar a criação de imagens mentais como mediadores da memorização e o uso de grupos de estudo em substituição às aulas didáticas sobre as estratégias (West, Welch \& Yassuda, 2000). Grupos de pesquisadores estão investigando a eficácia de treinos feitos através do computador (Guenther, Schaefer, Holzner, \& Kemmler, 2003; Laurance et al., 2002), sugerindo que este pode ser uma ferramenta importante na otimização da memória. Ainda entre os estudos inovadores, Dunlosky, Kubat-Silman e Hertzog (2003) descobriram que o treino de memória torna-se mais eficaz quando o idoso é ensinado a monitorar o processo de memorização, aprendendo a se auto-testar enquanto estuda a informação alvo, para descobrir os itens que necessitam ser re-estudados.

Tradicionalmente, nos estudos sobre treino, os idosos aprendem estratégias que podem melhorar o desempenho em tarefas de memória episódica, como a memorização de pares de faces e nomes ou listas de palavras, com possível aplicação no cotidiano. Atualmente, pesquisadores têm voltado sua atenção para a possibilidade de treino da memória operacional, considerada por autores como Salthouse (1991), como recurso cognitivo essencial para o bom funcionamento dos outros sistemas de memória, entre eles a memória episódica (Edwards et al., 2002; Wood et al., 2000).

Menciona-se ainda como inovação recente a realização de estudo de treino multicêntrico envolvendo um grande número de participantes: 2.832 pessoas entre 65 e 94 anos de idade (Ball et al., 2002). Participantes receberam um dos seguintes tipos de intervenção cognitiva: (a) treino de memória episódica verbal (grupo 1); (b) treino de raciocínio lógico (grupo 2); (c) treino de velocidade de processamento (grupo 3); e, (d) grupo controle (grupo 4). Participantes receberam 10 sessões de treino, e $60 \%$ da amostra recebeu quatro sessões de reforço 11 meses depois. Os resultados indicaram que $87 \%$ dos participantes do grupo 3 apresentaram desempenho melhor em tarefas de velocidade de processamento, $74 \%$ dos participantes do grupo 2 melhoraram em raciocínio lógico, e $26 \%$ dos participantes do grupo 1 melhoraram em memória. As sessões de reforço foram eficazes para a manutenção da velocidade de processamento e para o raciocínio lógico, mas não para memória. Os efeitos positivos do treino não foram detectados no dia-a-dia dos indivíduos, entretanto, efeitos positivos do treino se mantiveram presentes após dois anos, sugerindo que os efeitos destas intervenções podem ser duradouros.

Apesar de representar tema amplamente estudado, diversas questões empíricas referentes ao treino de memória no envelhecimento encontram-se sem resposta. Uma das principais diz respeito aos mecanismos que explicam o aumento na capacidade de memorizar dos participantes após o treino. Alguns autores (e.g., Yesavage, 1983; Yesavage, Lapp, \& Sheikh, 1989) sugerem que os participantes memorizam melhor após a intervenção, pois usam as técnicas divulgadas durante o treino. Surpreendentemente, a grande maioria dos estudos não incluiu uma avaliação objetiva da utilização das técnicas (ou estratégias) no pós-teste (West, 1995; West et al., 2000). É possível que outros fatores possam explicar o melhor desempenho dos participantes, tais como maior rapidez no processamento das informações, melhor atenção, ou menor ansiedade. Existe ainda a possibilidade do participante idoso intensificar o uso de uma estratégia que já usava anteriormente, como sugere estudo de Verhaeghen e Marcoen (1996).

Para estabelecer o uso de estratégias como mecanismo explicativo, a utilização das estratégias de memória deve ser medida objetivamente antes e depois da intervenção, um grupo controle que somente completa as avaliações deve ser incluído (para avaliar os efeitos da prática com atividades de memória), e deve-se avaliar a correlação entre o uso das estratégias e o desempenho nos testes de memorização no pré e no pós-teste. Poucos estudos cumpriram estas exigências (ver Saczynski, Willis, \& Schaie, 2000; Verhaeghen, \& Marcoen, 1996, como exceções).

Assim, as evidências a favor das estratégias como mecanismo responsável pelo ganho gerado pelo treino são até o 
momento preliminares. Outros mecanismos explicativos devem ser examinados para que os componentes essenciais das intervenções de memória sejam determinados. Por exemplo, é possível que alterações na constelação de crenças sobre a memória e o envelhecimento após o treino influenciem o desempenho.

A questão das crenças e atitudes sobre a memória e sua contribuição para as intervenções de memória constitui tema insuficientemente explorado na literatura. Diversos estudos investigaram o impacto do treino mnemônico sobre as crenças, supondo que o melhor desempenho talvez levasse a crenças mais positivas, e outros fizeram o caminho inverso, isto é, avaliaram a importância de alterar crenças negativas sobre memória e envelhecimento para potencializar os efeitos do treino. Os resultados destes estudos são controversos, como encontra-se relatado a seguir.

Zarit et al. (1981) e Scogin, Storandt e Lott (1985) documentaram que a melhora objetiva no desempenho de memória não leva a um menor número de queixas, e em consonância com outros estudos (Best, Hamlett \& Davis, 1992; Hill, Sheik \& Yesavage, 1987; Rebok \& Balcerak, 1989) concluíram que a melhora objetiva da memória não se traduz em auto-avaliações mais positivas. Outras pesquisas indicaram que intervenções que focam as crenças e as estratégias mnemônicas podem melhorar simultaneamente a memória objetiva (desempenho) e subjetiva (crenças e atitudes) (Caprio-Prevette \& Fry, 1996; Dittmann-Kohli, Lachman, Kliegl \& Baltes, 1991; Troyer, 2001; West, Bramblett, Welch, \& Bellott, 1992). Em estudo meta-analítico, Floyd e Scogin (1997) concluíram que o treino de memória tem maior efeito sobre o desempenho de memória do que sobre as crenças, sugerindo que a memória subjetiva é mais resistente a alterações. Finalmente, outros estudos documentaram que às vezes o idoso altera positivamente suas crenças sobre a memória, mas este fato não repercute no desempenho de memória (West et al., 2000).

Yassuda (1999), por exemplo, analisou o impacto da alteração das crenças na eficácia do treino de memória. Neste estudo, os participantes idosos receberam um dos seguintes treinos: treino mnemônico aliado a um componente educativo visando alterar crenças negativas sobre memória e envelhecimento, treino mnemônico somente e grupo controle, que recebeu treino após as avaliações. Os resultados revelaram que ao final da intervenção o primeiro grupo (crenças + estratégias) apresentou maior conhecimento e crenças mais positivas sobre a memória, quando comparado aos outros grupos. Entretanto, o desempenho em tarefas de memória episódica foi similar para os dois primeiros grupos, o que sugere que alterações na memória subjetiva (crenças e atitudes) não tornam o treino mais eficaz, em consonância com estudo anterior (Lachman, Weaver, Bandura, Elliott \& Lewkowicz, 1992). Desta forma, a alteração de crenças negativas ainda não tem suporte na literatura como mecanismo explicativo para o benefício do treino sobre o desempenho.

$\mathrm{Na}$ literatura, as crenças e atitudes sobre a memória são entendidas como elementos constituintes do conceito mais amplo de metamemória, que engloba conhecimentos, afetos e crenças sobre a memória (Welch \& West, 1995; Yassuda, 2004). As crenças são freqüentemente avaliadas através de questionários de queixas de memória ou de auto-eficácia. A auto-eficácia, que é a avaliação do indivíduo a respeito de sua capacidade para realizar determinadas ações (Bandura, 1997), vem sendo considerada como uma variável de muita importância para o desempenho cognitivo, e é possível que esta variável seja alterada pelo treino ou que esteja implicada nos efeitos positivos do mesmo (West et al., 2000).

Outras variáveis também podem estar associadas ao ganho após treino, como por exemplo, o aumento na velocidade de processamento de informações. A velocidade de processamento é freqüentemente caracterizada na literatura como recurso básico necessário ao bom funcionamento da memória (Salthouse, 1991). É possível que após o treino os idosos se tornem processadores mais rápidos e que isto contribua para o desempenho de memória no pós-teste. A manipulação experimental da velocidade de processamento em idosos já foi demonstrada experimentalmente em estudos de treino (e.g., Ball et al., 2002).

Menciona-se ainda a importância de fatores afetivos para o desempenho cognitivo e a necessidade destas variáveis serem mensuradas em estudos de treino. É notória a associação entre estados depressivos e o desempenho cognitivo (e.g., Scogin et al., 1985). Assim, é plausível supor que uma diminuição em sintomas depressivos originada pela participação em sessões de treino grupais seja um dos mecanismos responsáveis pelo melhor desempenho após treino.

No Brasil, o estudo sobre treino cognitivo no envelhecimento encontra-se em seus estágios iniciais e recebe tímida atenção dos pesquisadores. $\mathrm{O}$ bom funcionamento da memória é vital para que o idoso possa continuar a viver de maneira independente. Estudo epidemiológico longitudinal realizado em São Paulo que segue idosos há mais de 10 anos (Ramos, 2003) revelou alguns fatores que influenciaram o risco de morte dos participantes (determinados através de modelo multivariado de análise com regressão logística): sexo, idade, hospitalização prévia e positividade nos rastreamentos para déficit cognitivo e dependência no dia-a-dia. Como afirma Ramos, os únicos fatores mutáveis que poderiam diminuir o risco de morte seriam o estado cognitivo e o grau de dependência. Assim, conclui-se que intervenções de memória podem contribuir para a promoção da saúde e autonomia dos idosos, sendo os estudos sobre treino de memória amplamente justificados.

Outros estudos epidemiológicos brasileiros documentam que aproximadamente $7 \%$ dos idosos preenchem critérios para demência (Herrera, Caramelli \& Nitrini, 1998, 2002) ou apresentam deterioração cognitiva (Cerqueira, 2003). Estes estudos indicam que o declínio cognitivo constitui-se em um importante problema de saúde pública, considerando-se a dependência e o ônus familiar que acarreta. Estes dados reforçam a relevância das intervenções de memória, sejam elas treino ou reabilitação cognitiva (Bottino et al., 2002).

O presente estudo teve como objetivo testar os efeitos de um programa de treino de memória episódica de quatro sessões em uma amostra de brasileiros acima de 60 anos. 
Objetivou-se quantificar alterações entre o pré e o pós-teste em tarefas de memória episódica, e em variáveis que pudessem estar associadas ao desempenho de memória, tais como o uso de estratégias, as queixas de memória, a autoeficácia, a velocidade do processamento, e o humor. Especificamente, testou-se a hipótese que após o treino o idoso tem melhor desempenho de memória devido a aumento no uso das estratégias mnemônicas ensinadas ou devido a alterações nas variáveis supra citadas.

\section{Métodos}

\section{Participantes}

69 participantes foram recrutados para participar de uma pesquisa sobre memória em dois centros de convivência para a terceira idade. Os participantes eram idosos independentes residentes na comunidade que freqüentavam estes centros para realizar atividades físicas e sociais. Os participantes foram aleatoriamente subdivididos em grupo experimental (GE) e grupo controle (GC), cada um com três lotes de 12 participantes aproximadamente. Foram 35 participantes GE e 34 GC. Os participantes foram sub-divididos em pequenos grupos para as sessões de treino para facilitar a interação.

A Tabela 1 apresenta os dados sócio-demográficos da amostra. Comparações estatísticas realizadas entre os grupos GE e GC com o teste de Mann-Whitney revelaram que os grupos eram estatisticamente semelhantes quanto às variáveis sócio-demográficas. O teste Mann-Whitney foi usado porque a maioria das variáveis não apresentou distribuição normal.

Como o objetivo do estudo era avaliar os efeitos do treino de memória no envelhecimento normal, os participantes foram pré-avaliados para detectar a presença de depressão e de demência. Para avaliar depressão a Escala de Depressão Geriátrica ([GDS], Yesavage et al., 1983) foi utilizada, tendo como ponto de corte pontuação superior a cinco. $\mathrm{O}$ Mini-Exame do Estado Mental ([MEEM], Bertolucci, Brucki, Campacci, \& Juliano, 1994) foi utilizado para detectar comprometimento cognitivo. Pontos de corte adequados ao nível educacional dos participantes foram utilizados. Cinco senhoras participaram do estudo, mas seus dados não foram incluídos nas análises, pois apresentaram GDS superior a cinco pontos. Três participantes faltaram em mais de duas sessões e seus dados não foram incluídos nas análises. Assim, o número de participantes incluídos nas análises foi 61 . A Tabela 1 apresenta o desempenho do GE e GC nas tarefas de memória episódica no pré-teste. Observa-se desempenho superior para o GE, entretanto, esta diferença não atingiu significância estatística.

A avaliação inicial incluiu uma pergunta sobre saúde percebida, na qual o participante deveria indicar a qualidade de sua saúde em uma escala Likert que variou entre 10 (saúde ruim) e 1 (saúde excelente). A variável saúde percebida tem recebido atenção especial de pesquisadores, pois além de se correlacionar significativamente com saúde objetiva, parece sintetizar uma complexa interação de fatores envolvidos no bem-estar no idoso, com alto valor preditivo de mortalidade (Mossey \& Shapiro, 1982; Ramos, 2003). Os dados da Tabela 1 sugerem que os participantes apresentavam boa saúde. Foram relatados os remédios tomados regularmente, assim como internações recentes.

\section{Procedimentos}

A intervenção de memória incluiu seis sessões. Para o GE a primeira e a última sessão foram utilizadas para o pré e o pós-teste respectivamente. As quatro sessões intermediárias foram utilizadas para o treino de memória. Foram realizadas duas sessões por semana de 90 minutos cada. Os participantes do GC realizaram o pré-teste e o pós-teste na mesma semana que o $\mathrm{GE}$, entretanto receberam as quatro sessões de treino após o pós-teste, por motivos éticos.

Cada sessão de treino foi dividida em três partes de aproximadamente 30 minutos cada. $\mathrm{Na}$ primeira parte, houve exposição teórica com abertura para discussão. No primeiro encontro, discutiu-se os sub-sistemas da memória, no segundo memória e envelhecimento, no terceiro as estratégias externas, como o uso de calendários e agendas, e no quarto as estratégias internas, como a categorização. A segunda parte de cada sessão envolveu a execução de exercícios de atenção e de memória operacional, e a terceira parte compreendeu a prática da tarefa alvo: o uso de grifos em textos e categorização de listas (praticados alternadamente a cada sessão). As listas e textos utilizados no treino fazem parte dos materiais propostos em Yassuda, Lasca e Neri (2005) descritos a seguir. As listas e textos utilizados no treino foram diferentes daqueles usados no pré e pós-teste. O protocolo utilizado para guiar cada sessão está resumidamente descrito no Anexo A. Ele foi repetido de maneira exata em todos os grupos.

A cada sessão os participantes realizaram leituras em casa para se preparar para o próximo encontro. Para esta leitura foi utilizado o livro Deu Branco (Alvarez, 2002). Uma cópia do livro foi emprestada a cada participante. A cada sessão estes recebiam uma folha com os números das páginas a serem lidas e duas questões para guiar a leitura. O nível de aderência ao programa foi elevado, pois poucos participantes faltaram ou deixaram de realizar as tarefas de casa.

Em resumo, o procedimento envolveu os seguintes passos:

1. Pré avaliação individual para obtenção de dados sócio-demográficos e verificação dos critérios de exclusão;

2. Sessão do pré-teste para os grupos GE e GC;

3. Realização das quatro sessões de treino para o grupo GE;

4. Realização do pós-teste para os grupos GE e GC;

5. Realização das quatro sessões de treino para o grupo GC por motivos éticos.

\section{Materiais}

O pré e pós-teste incluíram avaliações de memória episódica, uso de estratégias de memória, velocidade de processamento das informações, metamemória (queixas de 
memória e auto-eficácia para memória) e humor. Estas variáveis foram estudadas, pois poderiam explicar os benefícios do treino de memória.

\section{Testes de memória episódica.}

Para avaliar a memória episódica no pré e pós-teste foram utilizadas duas listas de supermercado e dois textos. Estas tarefas foram selecionadas, pois possibilitam a avaliação do uso de estratégias, um dos objetivos do presente estudo. Além disto, já foram exaustivamente utilizadas em estudos internacionais anteriores e representam desafios de memória que fazem parte do cotidiano das pessoas idosas.

No pré e pós-teste foram utilizadas versões diferentes (B e C), mas equivalentes das listas de supermercado e dos textos para minimizar os efeitos de re-teste. Cada grupo GE e GC no pré e no pós-teste utilizou um número equivalente das versões $\mathrm{B}$ e $\mathrm{C}$ das listas e textos distribuídos entre os participantes. Os participantes que memorizaram a lista e o tex to $\mathrm{B}$ no pré-teste, receberam a lista e o tex to C no pósteste, e vice-versa.

As listas de supermercado usadas nas avaliações e treino, no total 4 versões diferentes mas equivalentes em seu grau de dificuldade, foram inicialmente desenvolvidas no laboratório da Dra. Robin West na Universidade da Flórida, e estudos-piloto estabeleceram sua equivalência em nível de dificuldade, facilidade para categorização, e popularidade dos itens (ver West, \& Thorn, 2001, para maiores detalhes sobre o processo de elaboração das listas). No Brasil, estas listas foram traduzidas para o português e algumas substituições nos itens foram realizadas para que pudessem conter itens populares dos nossos supermercados. Posteriormente, as listas foram avaliadas por três juizes especialistas e a equivalência das quatro versões foi testada em uma amostra composta por jovens e idosos (Yassuda et al., 2005).

Durante a memorização da lista, o participante teve três minutos para estudá-la e posteriormente mais três minutos para escrever os itens recordados em uma outra folha. A variável de interesse foi o número total de itens recordados.

Os textos usados no treino e nas avaliações foram selecionadas do manual desenvolvido por Dixon, Hultsch, e Hertzog (1989) - "Manual of twenty-five three tiered structurally equivalent text for use in aging research." Estes quatro textos (A e D foram usados no treino e $\mathrm{B}$ e $\mathrm{C}$ nas avaliações) são equivalentes quanto à estrutura semântica, com um número comparável de proposições, idéias importantes e detalhes. Estes textos foram traduzidos e adaptados para o português, avaliados por juizes especialistas, e a equivalência dos textos foi testada em estudo anterior (Yassuda et al., 2005). Os textos $\mathrm{B}$ e $\mathrm{C}$ foram usados em quantidade equivalente nos $\mathrm{GE}$ e $\mathrm{GC}$, seguindo o mesmo procedimento das listas explicado acima.

Durante a realização da tarefa, os participantes tiveram cinco minutos para ler o tex to impresso em uma folha branca, e posteriormente mais cinco minutos para escrever tudo o que lembraram sobre o mesmo em uma outra folha. A variável de interesse foi o número total de proposições recordadas.

\section{Uso de estratégias de memória.}

$\mathrm{O}$ uso de estratégias foi avaliado diretamente no protocolo do pré e do pós-teste. Na recordação das listas de supermercado foi calculada a medida de categorização de Bousfield (1953) utilizada frequientemente em estudos sobre estratégias de memória envolvendo listas (West, \& Thorn, 200 1; no Brasil ver Mello, 2003). Para a obtenção deste índice, calcula-se uma taxa de repetição intra-categórica (ratio of repetition), seguindo a fórmula $[\mathrm{RR}=\mathrm{r} / \mathrm{n}-1]$, onde $r$ representa o número de itens de uma mesma categoria que são recordados juntos e $\mathrm{n}$ o total de itens recordados. Este índice varia de 0 (nenhuma categorização) a 1 (categorização total).

Para cada tipo de lista, é importante calcular o índice de categorização que poderia ocorrer ao acaso, sem nenhum esforço do memorizador. Este índice consiste na divisão entre o número de itens abrangidos em cada categoria menos 1 (no caso destas listas $=5-1$ ) pelo total de itens presentes na lista, também menos 1 (neste caso 35-1). Assim, o índice correspondente ao nível de agrupamento categórico ao acaso corresponde a 0,12 para as lista adotadas neste estudo, pois 5-1/35-1 =0,12. Este índice indica que taxas de repetição intra-categórica acima de 0,12 representam taxas superiores ao agrupamento ao acaso, e representam um esforço do participante de categorizar a lista. Como informa a Tabela 2, mesmo no pré-teste o índice de categorização de Bousfield encontrou-se acima desta marca, o que sugere que alguns participantes já usavam a estratégia de categorização.

Para a recordação de textos, a medida de uso de estratégias foi o número de palavras grifadas pelos participantes no pré e no pós-teste, semelhante ao sistema utilizado por Saczynski et al. (2000). Cabe ressaltar que durante a leitura dos textos no pré e no pós-teste os participantes tinham lápis disponível e as instruções indicavam que o tex to poderia ser marcado. Entretanto, o uso de grifos não foi incentivado verbalmente pelo pesquisador durante o pré ou pós-teste.

\section{Velocidade de processamento.}

Para avaliar a velocidade de processamento de informações dos participantes, foi usado o sub-teste de transferência de símbolos da bateria de inteligência WAIS-R (Lezak, 1995), utilizado como uma medida de velocidade de processamento em diversas pesquisas anteriores (Salthouse, 1990, 1991). Em 90 segundos o participante copia os símbolos de uma legenda (que associa números a símbolos) embaixo de uma seqüência de números impressos no protocolo. A variável de interesse é o número de símbolos copiados.

\section{Metamemória.}

A metamemória foi avaliada através de dois questionários o MAC-Q e o MSEQ. O MAC-Q (Crook, Feher \& Larabee, 1992) avalia a presença de queixas de memória e já foi usado em nosso meio (Mattos et al., 2003). O MAC-Q inclui cinco questões que avaliam diferentes aspectos da memó- 
ria (memória para nomes de pessoas, números de telefone, onde colocou objetos, notícias de jornal, itens a serem comprados em lojas) e uma questão de apreciação geral da memória, comparando o momento presente a quando a pessoa tinha 18 a 20 anos. Os sujeitos devem escolher uma dentre cinco opções, variando entre "muito melhor agora" a "muito pior agora."

O MSEQ - Memory Self-Efficacy Questionaire (Berry, West \& Dennehey, 1989), que avalia a auto-eficácia relacionada à memória, foi usado no pré e no pós-teste, para captar alterações nas crenças dos participantes sobre a sua própria memória. O MSEQ foi traduzido e adaptado para o nosso meio, e suas características psicométricas junto à população brasileira foram apresentadas em outra publicação (Yassuda et al., 2005).

\section{Humor}

Para verificar se alterações no humor, potencialmente geradas pelo treino, poderiam estar associadas às mudanças no desempenho de memória, o GDS (Yesavage et al., 1983) foi reaplicado no pós-teste.

\section{Resultados}

Após a conclusão do treino, foram calculados os escores para as variáveis no pré e no pós-teste. Os protocolos da memorização de tex to foram conferidos por dois pesquisadores diferentes, que se familiarizaram com a estrutura dos textos e posteriormente contaram quantas idéias estavam contidas em cada protocolo. $30 \%$ dos protocolos foi aferido por ambos pesquisadores, e o grau de concordância entre os dois foi calculado, estando acima de $95 \%$. O restante dos protocolos foi aferido por somente um dos pesquisadores devido ao alto grau de concordância. Durante a fase de aferição e digitação dos dados os pesquisadores não sabiam a que grupo os participantes pertenciam, pois os protocolos foram identificados através de códigos numéricos.

Os dados foram analisados através do programa estatístico SAS System for Windows, versão 6.12., e o nível de significância adotado para os testes estatísticos foi de $5 \%$.

Foram realizadas análises de variância (ANOVA) para medidas repetidas que examinaram a interação entre os fatores Grupo (GE x GC) e Tempo (pré-teste x pós-teste). Interações que atingiram significância estatística ou se aproximaram dela foram analisadas em seguida através das comparações entre os pares de médias com o teste de Tukey. Foram realizados: teste de homogeneidade de variância, estimativa da magnitude do efeito e estimativa do poder estatístico.

Também foram calculadas diferenças percentuais delta (escore do pós-teste menos o escore do pré-teste) para todas as variáveis dependentes. Este delta representa o ganho associado ao treino documentado entre o pré e o pós-teste. Posteriormente, os deltas dos grupos GE e GC foram comparados através do teste de Mann-Whitney, devido à ausência de distribuição normal. A análise dos deltas foi realizada porque existe discordância na literatura a respeito da melhor maneira de medir os efeitos do treino. Alguns autores afir- mam que a comparação entre médias grupais (GE x GC) pode mascarar a melhora de alguns participantes, que seria captada através do cálculo dos deltas (para uma discussão mais detalhada sobre este tema ver Willis, 1987).

Finalmente, correlações de Spearman entre as múltiplas variáveis do estudo foram calculadas no pré e no pós-teste, para avaliar se o padrão de associação entre as variáveis seria alterado em função do treino.

A Tabela 1 apresenta os dados sócio-demográficos (ver Participantes). A Tabela 2 apresenta as médias e desvios padrão dos grupos GE e GC no pré e no pós-teste e a Tabela 3 apresenta os resultados das ANOVAS para medidas repetidas, que compararam os escores das variáveis dependentes entre os grupos e entre os tempos de avaliação.

Os resultados das ANOVAS e dos testes de Tukey, apresentados na Tabela 3, indicaram que algumas variáveis permaneceram inalteradas entre o pré e o pós-teste para ambos os grupos, como a memorização de listas, o GDS e o MSEQ. Algumas variáveis, como a velocidade de processamento (Símbolos) e queixas (MAC-Q) se alteraram na mesma direção em ambos os grupos: os participantes dos grupos GE e GC apresentaram-se mais rápidos no pós-teste, e com menor número de queixas de memória.

Para memorização de texto, observou-se ganho superior para o grupo experimental. O efeito da interação Grupo versus Tempo aproximou-se da significância estatística e o teste de Tukey revelou incremento superior para o GE. O mesmo padrão de resultados foi observado para a medida de categorização de Bousfield. Observou-se maior ganho no índice de categorização para o GE, apesar da interação entre os fatores Grupo x Tempo também ter somente se aproximado da significância estatística. Deve-se ressaltar que estes resultados revelam efeitos modestos do treino, considerando que o efeito da interação entre os fatores Grupo e Tempo somente aproximou-se da significância estatística.

Para a variável grifo, a interação Grupo versus Tempo foi estatisticamente significativa, assim como foi significativo o efeito do fator Tempo e Grupo. A comparação entre as médias indicou maior aumento na utilização de grifos para o GE do pré para o pós-teste.

Tabela 1

Média e Desvio Padrão (entre parênteses) para as Características Socio-Demográficas dos Participantes

\begin{tabular}{lrrrr}
\hline & Experimental & \multicolumn{2}{c}{ Controle } & p-valor \\
\hline Idade & $68,86(6,64)$ & 69,15 & $(5,22)$ & 0,90 \\
Escolaridade & $8,40(3,97)$ & 7,26 & $(4,08)$ & 0,20 \\
Renda & $5,94(5,90)$ & 5,62 & $(5,47)$ & 0,94 \\
Saúde & $3,12(1,77)$ & 2,68 & $(1,20)$ & 0,49 \\
Lista pré-teste & $12,62(4,40)$ & 10,91 & $(4,58)$ & 0,14 \\
Texto pré-teste & $20,91(9,61)$ & 16,62 & $(7,13)$ & 0,06 \\
\hline
\end{tabular}

Nota: $\mathrm{N}=$ A escolaridade foi avaliada em anos completos de educação formal, a renda foi avaliada em números de salários mínimos recebidos e a saúde foi avaliada por uma escala Likert que variou de 1 a 10, 1 sendo boa saúde e 10 saúde péssima. P-valor referente ao teste de Mann-Whitney para comparação entre grupos. 
Tabela 2

Média e Desvio Padrão (entre parênteses) para as Variáveis Dependentes

\begin{tabular}{|c|c|c|}
\hline Grupo & Pré-teste & Pós-teste \\
\hline & \multicolumn{2}{|c|}{ Memorização do texto } \\
\hline Experimental & $20,91 \quad(9,61)$ & $24,44 \quad(9,73)$ \\
\hline \multirow[t]{2}{*}{ Controle } & $16,62 \quad(7,13)$ & $18,00 \quad(7,63)$ \\
\hline & \multicolumn{2}{|c|}{ Memorização da lista } \\
\hline Experimental & $12,62(4,40)$ & $13,47 \quad(5,60)$ \\
\hline \multirow[t]{2}{*}{ Controle } & $10,91 \quad(4,58)$ & $11,71 \quad(4,52)$ \\
\hline & \multicolumn{2}{|c|}{ Número de grifos (texto) } \\
\hline Experimental & $7,88(12,95)$ & $17,91(12,17)$ \\
\hline \multirow[t]{2}{*}{ Controle } & $5,88 \quad(9,45)$ & $6,34 \quad(9,32)$ \\
\hline & \multicolumn{2}{|c|}{ Medida de Bousfield (lista) } \\
\hline Experimental & $0,30 \quad(0,23)$ & $0,48 \quad(0,23)$ \\
\hline \multirow[t]{2}{*}{ Controle } & $0,24 \quad(0,19)$ & $0,32 \quad(0,23)$ \\
\hline & \multicolumn{2}{|c|}{ Sub-teste símbolos WAIS-R } \\
\hline Experimental & $30,82(13,36)$ & $37,56(15,75)$ \\
\hline \multirow[t]{2}{*}{ Controle } & $29,56(14,01)$ & $34,31(15,17)$ \\
\hline & \multicolumn{2}{|c|}{ GDS } \\
\hline Experimental & $2,49 \quad(1,50)$ & $2,72 \quad(1,71)$ \\
\hline \multirow[t]{2}{*}{ Controle } & $2,24 \quad(1,28)$ & $2,72 \quad(1,53)$ \\
\hline & \multicolumn{2}{|c|}{ MAC-Q } \\
\hline Experimental & $26,32 \quad(4,62)$ & $22,68 \quad(6,50)$ \\
\hline \multirow[t]{2}{*}{ Controle } & $27,09 \quad(2,75)$ & $25,45 \quad(3,60)$ \\
\hline & \multicolumn{2}{|c|}{ MSEQ nível } \\
\hline Experimental & $2,54 \quad(0,81)$ & $2,43 \quad(1,02)$ \\
\hline \multirow[t]{2}{*}{ Controle } & $2,57(0,60)$ & $2,48 \quad(0,59)$ \\
\hline & \multicolumn{2}{|c|}{ MSEQ força } \\
\hline Experimental & $39,35(16,96)$ & $38,73(19,75)$ \\
\hline Controle & $42,02 \quad(9,58)$ & $39,60(10,90)$ \\
\hline
\end{tabular}

Nota: GE $\mathrm{n}=32, \mathrm{GC}$ n=29.
A Tabela 4, na próxima página, apresenta os resultados do teste de homogeneidade de variância, a estimativa da magnitude do efeito e a estimativa do poder estatístico, calculados no pré e pós-teste. Nota-se maior efeito e poder estatístico no pós-teste, pois as diferenças entre os grupos GE e GC são acentuadas após o treino.

Foram calculadas as diferenças percentuais (delta) para as variáveis dependentes entre os tempos pré e pós e foram realizadas comparações entre os deltas dos grupos. Houve diferença significativa apenas para a variável grifo $(p=0.003)$, sendo o delta do grupo GE maior que o do grupo GC. Como estes resultados foram semelhantes aos das ANOVAS, estes não serão apresentados.

Foram realizadas análises de regressão linear para os deltas da memorização do tex to e da memorização da lista em função das variáveis explicativas de interesse: grifos, categorização, símbolos, MSEQ, GDS, idade, escolaridade e saúde, para cada grupo separadamente. Primeiramente, foram ajustados os modelos univariados e posteriormente o modelo multivariado com critério stepwise de seleção de variáveis. Houve relação significativa apenas para a variável idade nos deltas tanto de memorização do texto $\left(\mathrm{p}=0,017, \mathrm{R}^{2}(\%)=18,16\right)$ como da lista $\left(\mathrm{p}=0,039, \mathrm{R}^{2}(\%)=\right.$ $13,86)$, com os participantes mais jovens do grupo GE apresentando maior ganho no desempenho do pré para o pósteste. Este resultado sugere que a idade é um fator preditivo de ganho associado ao treino de memória, com os participantes mais jovens se beneficiando mais da intervenção do que participantes mais idosos. O mesmo resultado não foi observado com relação à escolaridade.

Quanto às correlações de Spearman realizadas com os dados do pré-teste com os grupos GE e GC aglutinados, foram observadas correlações moderadas e significativas

Tabela 3

Resultados das ANOVAS comparando os Escores das Variáveis Dependentes entre os Grupos GE e GC no Pré e no Pós-Teste

\begin{tabular}{llcl}
\hline Variável & $\begin{array}{c}\text { Comparação entre } \\
\text { Grupos (GE x GC) }\end{array}$ & $\begin{array}{c}\text { Comparação entre } \\
\text { Tempos (Pré x Pós) }\end{array}$ & $\begin{array}{c}\text { Efeito da Interação } \\
\text { Grupos x Tempos }\end{array}$ \\
\hline GDS & $\mathrm{F}_{(1,59)}=0,19 ; \mathrm{p}=0,663$ & $\mathrm{~F}_{(1,59)}=2,37 ; \mathrm{p}=0,129$ & $\mathrm{~F}_{(1,59)}=0,94 ; \mathrm{p}=0,336$ \\
MAC-Q & $\mathrm{F}_{(1,58)}=3,31 ; \mathrm{p}=0,074$ & $\mathrm{~F}_{(1,58)}=21,13 ; \mathrm{p}<0,001^{\mathrm{a}}$ & $\mathrm{F}_{(1,58)}=2,16 ; \mathrm{p}=0,147$ \\
Grifos & $\mathrm{F}_{(1,59)}=9,05 ; \mathrm{p}=0,004$ & $\mathrm{~F}_{(1,59)}=11,60 ; \mathrm{p}=0,001$ & $\mathrm{~F}_{(1,59)}=8,40 ; \mathrm{p}=0,005^{\mathrm{b}}$ \\
Símbolos & $\mathrm{F}_{(1,59)}=0,30 ; \mathrm{p}=0,584$ & $\mathrm{~F}_{(1,59)}=22,45 ; \mathrm{p}<0,001^{\mathrm{c}}$ & $\mathrm{F}_{(1,59)}=1,91 ; \mathrm{p}=0,173$ \\
Texto & $\mathrm{F}_{(1,59)}=6,58 ; \mathrm{p}=0,013^{\mathrm{d}}$ & $\mathrm{F}_{(1,59)}=7,22 ; \mathrm{p}=0,009^{\mathrm{d}}$ & $\mathrm{F}_{(1,59)}=2,42 ; \mathrm{p}=0,125$ \\
Lista & $\mathrm{F}_{(1,58)}=2,54 ; \mathrm{p}=0,116$ & $\mathrm{~F}_{(1,58)}=1,75 ; \mathrm{p}=0,191$ & $\mathrm{~F}_{(1,58)}=0,01 ; \mathrm{p}=0,954$ \\
Categorização & $\mathrm{F}_{(1,59)}=5,07 ; \mathrm{p}=0,028^{\mathrm{e}}$ & $\mathrm{F}_{(1,59)}=15,26 ; \mathrm{p}<0,001^{\mathrm{e}}$ & $\mathrm{F}_{(1,59)}=2,74 ; \mathrm{p}=0,103$ \\
MSEQ Nível & $\mathrm{F}_{(1,59)}=0,06 ; \mathrm{p}=0,805$ & $\mathrm{~F}_{(1,59)}=0,95 ; \mathrm{p}=0,334$ & $\mathrm{~F}_{(1,59)}=0,01 ; \mathrm{p}=0,991$ \\
MSEQ Força & $\mathrm{F}_{(1,59)}=0,24 ; \mathrm{p}=0,629$ & $\mathrm{~F}_{(1,59)}=0,36 ; \mathrm{p}=0,550$ & $\mathrm{~F}_{(1,59)}=0,46 ; \mathrm{p}=0,502$ \\
\hline
\end{tabular}

Nota: $\mathrm{F}_{(\mathrm{a}, \mathrm{b})}=$ estatística $\mathrm{F}$ com “a” graus de liberdade no numerador e "b” graus de liberdade no denominador. $\mathrm{N}=61$.

a. Diferenças significativas: Pré $>$ Pós $(p<0,001)$, para ambos os grupos; b. Efeito significativo da interação, teste de Tukey GE: Pré<Pós (p<0,001); GC: Pré=Pós ( $p=0,628)$; c. Diferenças significativas: Pré $<$ Pós $(p<0,001)$, para ambos os grupos; d. Diferenças significativas, teste de Tukey GE: Pré<Pós ( $p=0,005)$; GC: Pré=Pós ( $p=0,437)$; e. Diferenças significativas, teste de Tukey GE: Pré $<$ Pós $(p<0,001)$; GC: Pré=Pós $(p=0,131)$. 
estatisticamente entre as medidas de desempenho (lista e texto) e a medida de categorização de Bousfield, a velocidade de processamento e a auto-eficácia. No pós-teste, quando as correlações foram calculadas separadamente para os grupos (com $n$ reduzido devido à separação dos grupos), o padrão dos resultados não foi claro e não confirmou a hipótese de maior associação entre estratégia e desempenho após o treino. Entretanto, notou-se maior associação entre as variáveis de desempenho e de auto-eficácia para ambos os grupos. Devido à limitação de espaço, estas correlações não serão apresentadas.

\section{Discussão}

O presente estudo investigou os efeitos de um programa de treino de memória de quatro sessões de 90 minutos oferecido a idosos brasileiros saudáveis. Foram avaliados diversos mecanismos que poderiam explicar benefícios do treino sobre a memória, como maior uso de estratégias, maior velocidade de processamento, melhor humor ou ainda crenças mais positivas sobre a memória.

Os resultados sugeriram efeitos modestos do treino. Houve um aumento significativo no uso de grifos durante a memorização do texto e um aumento modesto na categorização da lista para o GE no pós-teste superior ao GC. Foi documentada também modesta vantagem para o GE sobre o GC no pós-teste na memorização de texto. Este padrão de resultados sugere que uma intensificação no uso das estratégias pode ser o mecanismo responsável pela melhora na memorização após treino. Os outros mecanismos (além do uso de estratégias) que poderiam explicar os benefícios do treino sobre o desempenho de memória permaneceram inalterados após intervenção (GDS, MSEQ) ou tiveram o mesmo padrão de alteração para os dois grupos (símbolos e MAC-Q).

Os resultados para as variáveis de desempenho e estratégias confirmam a idéia já presente na literatura internacional que o ensino de estratégias mnemônicas não é um processo rápido quando o idoso para a usar e a se beneficiar de uma estratégia assim que a mesma lhe é apresentada. O ensino de estratégias de memória parece ser um processo gradual no qual inicialmente o participante não usa espontaneamente a estratégia (deficiência da produção); posteriormente passa a usar a estratégia, mas seu desempenho de memória não aumenta (deficiência da utilização);

Tabela 4

Comparação dos Escores entre Grupos e Tempos, através das ANOVAS

\begin{tabular}{|c|c|c|c|}
\hline Variável & Teste de Levene $*$ & $\begin{array}{c}\text { Estimativa da } \\
\text { Magnitude do Efeito }\end{array}$ & $\begin{array}{c}\text { Estimativa do } \\
\text { Poder Estatístico }\end{array}$ \\
\hline \multirow{2}{*}{ GDS } & Pré: $\mathrm{F}(1,59)=1.43 ; \mathrm{p}=0.236$ & Pré: $E=1.00$ & Pré: $14.0 \%$ \\
\hline & Pós: $\mathrm{F}(1,59)=0.52 ; \mathrm{p}=0.472$ & Pós: $\mathrm{E}=1.17$ & Pós: $3.0 \%$ \\
\hline \multirow{2}{*}{ MAC-Q } & Pré: $F(1,58)=2.44 ; p=0.124$ & Pré: $\mathrm{E}=2.76$ & Pré: $17.5 \%$ \\
\hline & Pós: $\mathrm{F}(1,58)=8.85 ; \mathrm{p}=0.004$ & Pós: $\mathrm{E}=3.83$ & Pós: $53.0 \%$ \\
\hline \multirow[t]{2}{*}{ Grifos } & Pré: $\mathrm{F}(1,59)=1.92 ; \mathrm{p}=0.172$ & Pré: $\mathrm{E}=7.90$ & Pré: $14.3 \%$ \\
\hline & Pós: $\mathrm{F}(1,59)=2.39 ; \mathrm{p}=0.128$ & Pós: $\mathrm{E}=7.80$ & Pós: $98.5 \%$ \\
\hline \multirow[t]{2}{*}{ Símbolos } & Pré: $\mathrm{F}(1,59)=0.66 ; \mathrm{p}=0.418$ & Pré: $\mathrm{E}=9.60$ & Pré: $4.0 \%$ \\
\hline & Pós: $\mathrm{F}(1,59)=0.05 ; \mathrm{p}=0.820$ & Pós: $\mathrm{E}=11.10$ & Pós: $12.6 \%$ \\
\hline \multirow[t]{2}{*}{ Texto } & Pré: $F(1,59)=0.87 ; p=0.355$ & Pré: $\mathrm{E}=6.10$ & Pré: $53.0 \%$ \\
\hline & Pós: $\mathrm{F}(1,59)=1.35 ; \mathrm{p}=0.250$ & Pós: $\mathrm{E}=6.30$ & Pós: $81.0 \%$ \\
\hline \multirow[t]{2}{*}{ Lista } & Pré: $F(1,58)=0.13 ; p=0.717$ & Pré: $\mathrm{E}=3.20$ & Pré: $35.0 \%$ \\
\hline & Pós: $\mathrm{F}(1,58)=1.90 ; \mathrm{p}=0.173$ & Pós: $\mathrm{E}=3.70$ & Pós: $27.0 \%$ \\
\hline \multirow[t]{2}{*}{ Categorização } & Pré: $\mathrm{F}(1,59)=0.81 ; \mathrm{p}=0.372$ & Pré: $\mathrm{E}=0.15$ & Pré: $17.0 \%$ \\
\hline & Pós: $\mathrm{F}(1,59)=0.01 ; \mathrm{p}=0.988$ & Pós: $\mathrm{E}=0.16$ & Pós: $77.0 \%$ \\
\hline \multirow[t]{2}{*}{ MSEQ Nível } & Pré: $\mathrm{F}(1,59)=1.31 ; \mathrm{p}=0.257$ & Pré: $\mathrm{E}=0.50$ & Pré: $4.5 \%$ \\
\hline & Pós: $\mathrm{F}(1,59)=4.01 ; \mathrm{p}=0.051$ & Pós: $\mathrm{E}=0.60$ & Pós: $4.0 \%$ \\
\hline \multirow{2}{*}{ MSEQ Força } & Pré: $\mathrm{F}(1,59)=3.05 ; \mathrm{p}=0.086$ & Pré: $\mathrm{E}=9.80$ & Pré: $11.5 \%$ \\
\hline & Pós: $\mathrm{F}(1,59)=6.22 ; \mathrm{p}=0.015$ & Pós: $\mathrm{E}=11.60$ & Pós: $4.0 \%$ \\
\hline
\end{tabular}

Nota: $\mathrm{F}(\mathrm{a}, \mathrm{b})=$ estatística F com "a" graus de liberdade no numerador e "b" graus de liberdade no denominador.

*Teste de homogeneidade de variância de Levene em cada tempo (Pré e Pós). 
e finalmente, o participante usa a estratégia espontaneamente e seu desempenho aumenta devido ao uso da estratégia (Bjorklund, Miller, Coyle \& Slawinski, 1997).

Os resultados apresentados sugerem que os idosos que participaram do treino intensificaram o uso das estratégias ensinadas, entretanto, o desempenho de memória não foi proporcionalmente impactado por este fato, o que sugere a presença da deficiência da utilização. Aparentemente, o treino com quatro sessões foi suficiente para encorajar os participantes a usar as estratégias, mas não foi suficiente para levá-los ao estágio no qual as estratégias já automatizadas se convertem em desempenho expressivamente melhor. Este padrão de resultados foi encontrado em estudos anteriores (West et al., 2000; Yassuda, 1999).

Em pesquisa anterior, Wood et al. (2000) demonstraram através de avaliações consecutivas realizadas após cada sessão de treino que a melhora no desempenho de memória se torna estatisticamente significativa a partir da quarta sessão, tanto para o treino que promoveu o uso de imagens mentais como para o treino que visou o aumento na velocidade de processamento. Dados do presente estudo estão em congruência com os dados de Wood et al., indicando modesta melhora no desempenho após quatro sessões de treino. Futuras pesquisas devem testar o efeito de treinos de memória mais prolongados para os idosos brasileiros, que tendem a ter menor escolaridade. Alguns estudos na literatura internacional (por exemplo, Stigsdotter-Neely, \& Backman, 1993, Ball et al., 2002) sugerem que o prolongamento do treino e a inclusão de múltiplos componentes podem gerar resultados mais eficazes. Futuros estudos também deveriam contemplar critérios de exclusão mais rígidos incluindo rastreamento mais abrangente das funções cognitivas, antes da admissão de idosos aos grupos de treino. Neste estudo somente o MEEM foi utilizado e é possível que tenha ocorrido grande variabilidade entre os participantes quanto às habilidades cognitivas. Participantes com declínio cognitivo além do esperado para a idade não captado pelo MEEM podem ter sido incluídos no presente estudo limitando os efeitos do treino.

Surpreendentemente, o treino de memória não alterou a variável auto-eficácia. Estudos anteriores (Best et al., 1992; Caprio-Prevette \& Fry, 1996; Troyer, 2001; West et al., 1992) relataram a possibilidade do treino alterar crenças e desempenho simultaneamente. É possível que as crenças e atitudes sobre memória e envelhecimento não tenham sido enfatizadas suficientemente durante o programa, apesar de terem sido abordadas através da leitura realizada. Entretanto, ressalta-se que após a intervenção ou simples retestagem (no caso dos GC) ocorreu maior associação entre as variáveis de desempenho e de auto-avaliação, no caso a auto-eficácia. A prática com tarefas de memória parece calibrar a capacidade do idoso de julgar seu desempenho em tarefas cognitivas.

Também contrário às expectativas, o treino em grupo não influenciou o humor dos participantes que manteve-se estável durante a intervenção nas duas condições experi- mentais. Este achado pode estar relacionado aos critérios de exclusão utilizados para este estudo, pois indivíduos com pontuação compatível com depressão não foram incluídos nas análises, fato que restringiu a variabilidade da variável humor.

Nota-se que para o grupo GC somente participar de sessões de avaliação, isto é, praticar com tarefas de memória, levou a uma redução nas queixas de memória e a um aumento na velocidade de processamento equivalente ao ocorrido com os GE. Estes resultados são relevantes, pois sugerem que reuniões grupais que ofereçam prática com tarefas de memória podem gerar benefícios aos idosos saudáveis. Estes resultados estão em congruência com estudo anterior (Dittmann-Kholi et al., 1991) e sugerem que mesmo sem instrução direta os idosos podem caminhar em direção ao seu desempenho máximo.

Outro resultado que merece destaque é o fato da idade ser preditiva da magnitude do ganho gerado pelo treino. Este resultado está em congruência com a meta-análise de Verhaeghen et al. (2002) e inúmeros outros estudos que apontam para os limites da plasticidade com o avançar da idade. Estudos de treino realizados com grupos de adultos jovens e idosos documentaram que os jovens tendem a apresentar maiores ganhos no desempenho do que os idosos, sugerindo maior plasticidade entre os jovens (Verhaeghen \& Marcoen, 1996).

Em síntese, no Brasil, as intervenções de memória para idosos saudáveis assim como a reabilitação cognitiva para idosos portadores de comprometimento cognitivo além do esperado para a idade compreendem temas pouco estudados. Deve-se ressaltar uma vez mais a relevância do tema, visto que a funcionalidade cognitiva do idoso está relacionada à sua saúde e qualidade de vida. $\mathrm{O}$ presente estudo revelou resultados modestos para uma intervenção de curta duração e indicou que o uso de estratégias pode estar relacionado aos ganhos de desempenho gerados pelo treino. Os próximos estudos devem testar a eficácia de diferentes tipos de intervenção, sempre visando a obtenção de resultados duradouros (manutenção) e que se generalizem para os desafios cognitivos diários dos nossos idosos.

\section{Referências}

Alvarez, A. M. M. (2002). Deu branco. São Paulo, SP: Best Seller. Backman, L. (1989). Varieties of memory compensation by older adults in episodic remembering. In L. W. Poon, D. C. Rubin \& B.C. Wilson (Eds.), Everyday cognition in adulthood and late life (pp. 509-544). Cambridge,UK: Cambridge University Press.

Ball, K., Berch, D. B., Helmers, K. F., Jobe, J. B., Leveck, M. D., Marsiske, M., \& Morris, J. et al. (2002). Effects of cognitive training interventions with older adults: A randomized controlled trial. Journal of the American Medical Association, 288(18), 2271-2281.

Baltes, P. B., \& Baltes, M. M. (1990). Psychological perspectives on successful aging: The model of selective optimization with compensation. In P. B. Baltes \& M. M. Baltes (Eds.), Successful 
aging: Perspectives from the behavioral sciences (pp. 1-34). Cambridge, UK: Cambridge University Press.

Bandura, A. (1997). Self-efficacy: The exercise of control. New York: W. H. Freeman.

Berry, J. M., West, R. L., \& Dennehey, D. M. (1989). Reliability and validity of the self-efficacy questionnaire. Developmental Psychology, 25, 701-713.

Bertolucci, P. H. F., Brucki, S. M. D., Campacci, S. R., \& Juliano, Y. (1994). O Mini-Exame do Estado Mental em uma população geral: Impacto da escolaridade. Arquivos de Neuro-Psiquiatria 52, 1-7.

Best, D. L., Hamlett, K. W., \& Davis, S. W. (1992). Memory complaint and memory performance in the elderly: The effects of memory-skills training and expectancy change. Applied Cognitive Psychology, 6, 405-416.

Bjorklund, D. F., Miller, P. H., Coyle, T. R., \& Slawinski, J. L. (1997). Instructing children to use memory strategies: Evidence of utilization deficiencies in memory training studies. Developmental Review, 17, 411-441.

Bottino, C. M. C., Carvalho, I., Alvarez, A. M. M., Ávila, R., Zukauskas, P. R., Bustamante, S. E. Z., \& Andrade, F. C. et al. (2002). Cognitive rehabilitation in Alzheimer's disease patients: Multidisciplinary team report. Arquivos de Neuropsiquiatria, 60, 70-79.

Bousfield, W. A. (1953). The occurrence of clustering in the recall of randomly arranged associates. Journal of General Psychology, 49, 229-240.

Caprio-Prevette, M. D., \& Fry, P.S. (1996). Memory enhancement program for community-based older adults: Development and evaluation. Experimental Aging Research, 22, 281-304.

Cerqueira, A. T. A. R. (2003). Deterioração cognitiva e depressão. In M.L. Lebrão \& Y. A. O Duarte (Eds.), O projeto SABE no município de São Paulo: Uma abordagem inicial (pp. 143-165). Brasília, DF: OPAS/OMS.

Crook T. H., Feher, E. P., \& Larabee, G. J. (1992). Assessment of memory complaint in age-associated memory impairment: The MAC-Q. International Psychogeriatrics, 4(2), 165-175.

Dittmann-Kohli, F., Lachman, M. E., Kliegl, R., \& Baltes, P. B. (1991). Effects of cognitive training and testing on intellectual efficacy beliefs in elderly adults. Journal of Gerontology, 46(4), 162-164.

Dixon, R. A., Hultsch, D. F., \& Hertzog, C. (1989). A manual of twenty-five three-tiered structurally equivalent text for use in aging research (CRGCA Tech. Rep. No. 2). Victoria, Canada: University of Victoria.

Dunlosky, J. \& Hertzog, C. (1998). Training programs to improve learning in later adulthood: Helping older adults educate themselves. In D. J. Hacker, J. Dunlosky \& A. C. Graesser (Eds.), Metacognition in educational theory and practice (pp. 249276). Mahwah, NJ: Erlbaum.

Dunlosky, J., Kubat-Silman, A. K.; Hertzog, C. (2003). Training monitoring skills improves older adults' self-paced associative learning. Psychology and Aging, 18(2), 340-345.

Edwards, J. D., Wadley, V. G., Meyers, R. S., Roenker, D. R., Cissell, G. M., \& Ball, K. K. (2002). Transfer of a speed of processing intervention to near and far cognitive functions. Gerontology, 48(5), 329-340.

Floyd, M., \& Scogin, F. (1997). Effects of memory training on subjective memory functioning and mental health of older adults: A meta-analysis. Psychology and Aging, 12(1), 150-161.

Guenther, V. K., Schaefer, P., Holzner, B. J., \& Kemmler, G. W. (2003). Long-term improvements in cognitive performance through computer-assisted cognitive training: A pilot study in a residential home for older people. Aging and Mental Health, $7(3)$, 200-206.

Herrera, E., Jr., Caramelli, P., \& Nitrini, R. (1998). Estudo epidemiológico de demência na cidade de Catanduva - Estado de São Paulo - Brasil. Revista de Psiquiatria Clínica, 25, 70-73.

Herrera, E., Jr., Caramelli, P., \& Nitrini, R. (2002). Epidemiologic survey of dementia in a community dwelling Brazilian population. Alzheimer's Disease Association, 18, 103-108.

Hill, R. D., Sheik, J. I., \& Yesavage, J. (1987). The effect of mnemonic training on perceived recall confidence. Experimental Aging Research, 13(4), 185-188.

Kausler, D. H. (1991). Experimental Psychology, cognition, and human aging (2nd. ed.). New York: Springer-Verlag.

Hulicka, I. M., \& Grossman, J. L. (1967). Age group comparisons for the use of mediators in paired-associate learning. Journal of Gerontology, 17, 360-367.

Lachman, M. E., Weaver, S. L., Bandura, M., Elliott, E., \& Lewkowicz, C. J. (1992). Improving memory and control beliefs through cognitive restructuring and self-generated strategies. Journal of Gerontology, 47(5), 293-299.

Lasca, V. B. (2003). Treinamento de memória no envelhecimento normal: Um programa aplicado a idosos. Tese de Mestrado nãopublicada, Universidade Estadual de Campinas, SP.

Laurance, H. E., Thomas, K. G. F., Newman, M. C., Kaszniak, A. W., Nadel, L., \& Jacobs, W. J. (2002) Older adults map novel environments but do not place learn: Findings from a computerized spatial task. Aging, Neuropsychology, and Cognition, 9(2), 85-97.

Lezak, M. (1995). Neuropsychological Assessment. New York: Oxford University Press.

Mattos, P., Lino, V., Rizo L., Alfano A., Araújo, C., \& Raggio, R (2003) Memory complaints and test performance in healthy elderly persons. Arquivos de Neuro-Psiquiatria, 61 (4), 920-924.

Mello, C. B. (2003). Estratégias categóricas de recordação e formação de conceitos em crianças de sete a quatorze anos de idade. Tese de Doutorado não-publicada, Universidade de São Paulo, SP.

Mossey, J. M. \& Shapiro, E. (1982). Self-rated health: A predictor of mortality among the elderly. American Journal of Public Health, 72, 800-808.

Ramos, L. R. (2003). Fatores determinantes do envelhecimento saudável em idosos residentes em centro urbano: Projeto Epidoso, São Paulo. Cadernos de Saúde Pública, 19, 793-797.

Rebok, G. W., \& Balcerak, L. J. (1989). Memory self-efficacy and performance differences in young and old adults: The effect of mnemonic training. Developmental Psychology, 25, 714-72 1.

Saczynski, J. S., Willis, S. L., \& Schaie, K.W. (2000). Strategy use and cognitive training effects. Poster presented at the Cognitive Aging Conference, Atlanta, Georgia.

Salthouse, T. A. (1990). Working memory as a processing resource in cognitive aging. Developmental Review, 10, 101-124.

Salthouse, T. A. (1991). Theoretical perspectives on cognitive aging. Hillsdale, NJ: Erlbaum.

Scogin, F., Storandt, M., \& Lott, L. (1985). Memory-skills training, memory complaints, and depression in older adults. Journal of Gerontology, 4O(5), 562-568.

Stigsdotter Neely, A., \& Backman, L. (1993). Long-term maintenance of gains from memory training in older adults: Two 3.5-year follow-up studies. Journal of Gerontology, 48(5), 233-237.

Stuart-Hamilton, I. (2002). A Psicologia do envelhecimento: Uma introdução. Porto Alegre, RS: Artmed. 
Troyer, A. K. (2001). Improving memory knowledge, satisfaction, and functioning via an education and intervention program for older adults. Aging, Neuropsychology, E Cognition, 8(4), 256-268.

Verhaeghen, P. (2000). The interplay of growth and decline: Theoretical and empirical aspects of plasticity of intellectual and memory performance in normal old age. In R. D. Hill, L. Backman, A. Stigsdotter-Neely (Eds.), Cognitive rehabilitation in old age (pp. 3-22). Oxford, UK: Oxford University Press.

Verhaeghen, P., \& Marcoen, A. (1996). On the mechanisms of plasticity in young and older adults after instruction in the method of loci: Evidence for an amplification model. Psychology and Aging, 11, 164-178.

Verhaeghen, P., Marcoen, A., \& Goosens, L. (1992). Improving memory performance in the aged through mnemonic training: A meta-analytic study. Psychology and Aging, 7(2), 242-251.

Welch, D. C., \& West, R. L. (1995). Self-efficacy and mastery: Its application to issues of environmental control, cognition, and aging. Developmental Review, 15, 150-171.

West, R. L. (1995). Compensatory strategies for Age-Associated Memory Impairment. In A. D. Baddeley, B. A. Wilson, \& F. N. Watts (Eds.), Handbook of memory disorders, (pp. 481-500). New York: John Wiley.

West, R. L., Bramblett, J. P., Welch, D. C., \& Bellott, B. (1992, April). Memory training for the elderly: An intervention designed to improve memory skills and memory evaluation. Paper presented at the Cognitive Aging Conference, Atlanta, GA.

West R. L., \& Thorn, R. M. (2001). Goal-setting, self-efficacy, and memory performance in older and younger adults. Experimental Aging Research, 27, 41-65.

West, R. L., Welch, D. C., \& Yassuda, M. S. (2000). Innovative approaches to memory training for older adults. In R. D. Hill, L. Backman, A. Stigsdotter-Neely (Eds.), Cognitive rehabilitation in old age (pp. 81-105). Oxford, UK: Oxford University Press.

Willis, S. L. (1987). Cognitive interventions in the elderly. In K. W. Schaie (Ed.), Annual review of gerontology and geriatrics (Vol. 7, pp. 159-188). New York: Springer.

Wood, G. M. O., Haase, V. G., Araújo, J. R., Scalioni, I. G., Lima, E. P. \& Sampaio, J. R. (2000). Desenvolvimento cognitivo adulto: A avaliação e a reabilitação da capacidade de memória de trabalho. In V. G. Haase, R. Rothe-Neves, C. Käppler, M. L. M. Teodoro \& G. M. O. Wood (Eds.), Psicologia do desenvolvimento: Contribuições interdisciplinares (pp. 121-144). Belo Horizonte, MG: Health.

Yassuda, M. S. (1999). Memory beliefs and memory training: The effects of an educational intervention. Tese de Doutorado nãopublicada, Universidade da Flórida, Gainesville, Estados Unidos da América.

Yassuda, M. S. (2006). Memória e envelhecimento saudável. In E. V. Freitas, L. Py, F. A. X. Cançado, J. Doll, M. L. Gorzoni, \& S. M. Rocha (Eds.), Tratado de Geriatria e Gerontologia (pp. 12451251). Rio de Janeiro, RJ: Guanabara Koogan.

Yassuda, M.S. (2004). Desempenho de memória e percepção de controle no envelhecimento saudável. In Neri, A. L. \& Yassuda, M. S. (Eds.), Velhice bem-sucedida: Aspectos afetivos e cognitivos. Campinas, SP: Papirus.

Yassuda, M. S, Lasca, V. B., \& Neri, A.L. (2005). Meta-memória e auto-eficácia: Um estudo de validação de instrumentos de pesquisa sobre memória e envelhecimento Psicologia: Reflexão e Crítica, 18(1), 78-90.

Yesavage, J. A. (1983). Imagery pretraining and memory training in the elderly. International Journal of Experimental and Clinical Gerontology, 29, 271-275.

Yesavage, J. A., Brink. T. L., Rose, T. L., Lum, O., Huang, V., Adey, M. B., \& Leirer, V. O. (1983). Development and validation of a depression screening scale: A preliminary report. Journal of Psychiatric Research, 17(1), 37-49.

Yesavage, J. A., Lapp, D., Sheikh, J. I. (1989). Mnemonics as modified for use by the elderly. In L. W. Poon, D. C. Rubin, \& B. C. Wilson (Eds.), Everyday cognition in adulthood and late life (pp. 573-597). Cambridge, UK: Cambridge University Press.

Zarit. S. H., Cole, K. D., \& Guider, R. L. (1981). Memory training strategies and subjective complaints of memory in the aged. The Gerontologist, 21, 158-164.
Recebido: $31 / 08 / 2004$ $1^{a}$ revisão: 20/01/2005 $2^{a}$ revisão: 07/11/2005 Aceite final: 23/03/2006 


\begin{abstract}
Anexo A
Programação das sessões de treino de memória

Sessão 1

Tarefa de casa: ler da página 17 a 31 do livro Deu Branco, e responder em folha separada:

1. Cite diferentes tipos de memória. Você pode dar exemplos de alguns?

2. Quais são as fases da memória?

Aula expositiva com abertura para discussão: as fases da memorização (atenção, gravação e recordação).

Exercício de atenção: participantes observam fotos individualmente por 3 minutos, depois em pares contam para o seu par tudo que foi visto na foto, agora não mais visível.

Aula expositiva com abertura para discussão: os sub-sistemas da memória (memória imediata, operacional, longa duração).

Exercício de memória operacional: colocar 3 palavras pronunciadas em ordem alfabética sem anotar.

Tarefa alvo: explicações sobre os benefícios do grifo para memorização de textos e posterior exercício de memorização de tex to com encorajamento para a utilização de grifos.
\end{abstract}

Sessão 2

Tarefa de casa: ler da página 33 a 39 e 51 a 61 do livro Deu Branco, e responder em folha separada:

1. Quais são as principais alterações na memória com a idade?

2. Dê exemplos de coisas que você pode fazer para melhorar sua memória.

Breve revisão da aula passada.

Aula expositiva com abertura para discussão: aspectos da memória que se alteram e aspectos que não se alteram ao longo do envelhecimento.

Exercício de atenção: seqüências de batidas na mesa que são transformadas em dígitos. Por exemplo: **** * ** se transforma em 3, 1, 2.

Exercício de memória operacional: participantes observam fragmentos de palavras na lousa (dit, m, o, su, osca) e mentalmente formam palavras sem usar o mesmo fragmento mais de uma vez (súdito, mosca), sem anotar.

Tarefa alvo: explicações sobre os benefícios da organização para a memória, explicações sobre como organizar uma lista de supermercado em categorias (oferecendo um exemplo) e posterior exercício de memorização de lista com encorajamento para utilização de categorização.

Sessão 3

Tarefa de casa: ler da página 63 a 74 do livro Deu Branco, e responder em folha separada:

1. Que estratégias internas você poderia usar para gravar o caminho para o seu novo médico?

2. Para guardar informações pessoais sobre um(a) novo(a) amigo(a)?

3. Para lembrar do nome de um restaurante?

Breve revisão da aula passada.

Aula expositiva com abertura para discussão: apresentação das técnicas mnemônicas externas (calendários, listas, alarmes, mudanças ambientais) e maneiras de usá-las de modo eficaz.

Exercício de atenção dividida: participantes observam padrão desenhado na lousa contendo diversos quadrados, círculos, triângulos e corações, e enquanto repetem uma seqüência silábica em voz alta (pa-pa-ra-pa-pa) devem contar quantas figuras de cada tipo estão desenhadas na lousa.

Exercício de memória operacional: participantes realizam operações matemáticas simples mentalmente colocadas na lousa $(8 \times 2,3 \times 4,7 \times 5)$ e depois somam os resultados parciais.

Tarefa alvo: explicações sobre os benefícios do grifo para memorização de textos e posterior exercício de memorização de tex to com encorajamento para a utilização de grifos.

Sessão 4

Tarefa de casa : ler da página 74 a 84 do livro Deu Branco, e responder em folha separada:

1. Para que serve a técnica da prática distribuída?

2. O que podemos fazer para recordar informações que estão na ponta da língua?

Breve revisão da aula passada.

Aula expositiva com abertura para discussão: apresentação das técnicas mnemônicas internas (associações verbais, imagens mentais, histórias) e maneiras de usá-las de modo eficaz.

Exercício de atenção: dominó fonológico, um participante fala uma palavra (boneca) e o seguinte deve iniciar sua palavra com a sílaba final da palavra do participante anterior (caneta).

Exercício de memória operacional: colocar palavras (adulto, bebê, adolescente) na seqüência temporal correta (bebê, adolescente, adulto). Ver Deu Branco para outros exercícios possíveis.

Tarefa alvo: explicações sobre os benefícios da organização para a memória, explicações sobre como organizar uma lista de supermercado em categorias (oferecendo um exemplo) e posterior exercício de memorização de lista com encorajamento para utilização de categorização.

Revisão do curso. 